Document downloaded from:

http://hdl.handle.net/10251/63303

This paper must be cited as:

Bockelt, AS.; Palací López, J.; Vidal Rodriguez, B. (2015). All-Fiber Centralized Architecture for Parallel Terahertz Censors. IEEE Transactions on Terahertz Science \&amp; Technology. 5(1):137-144. doi:10.1109/TTHZ.2014.2373313.

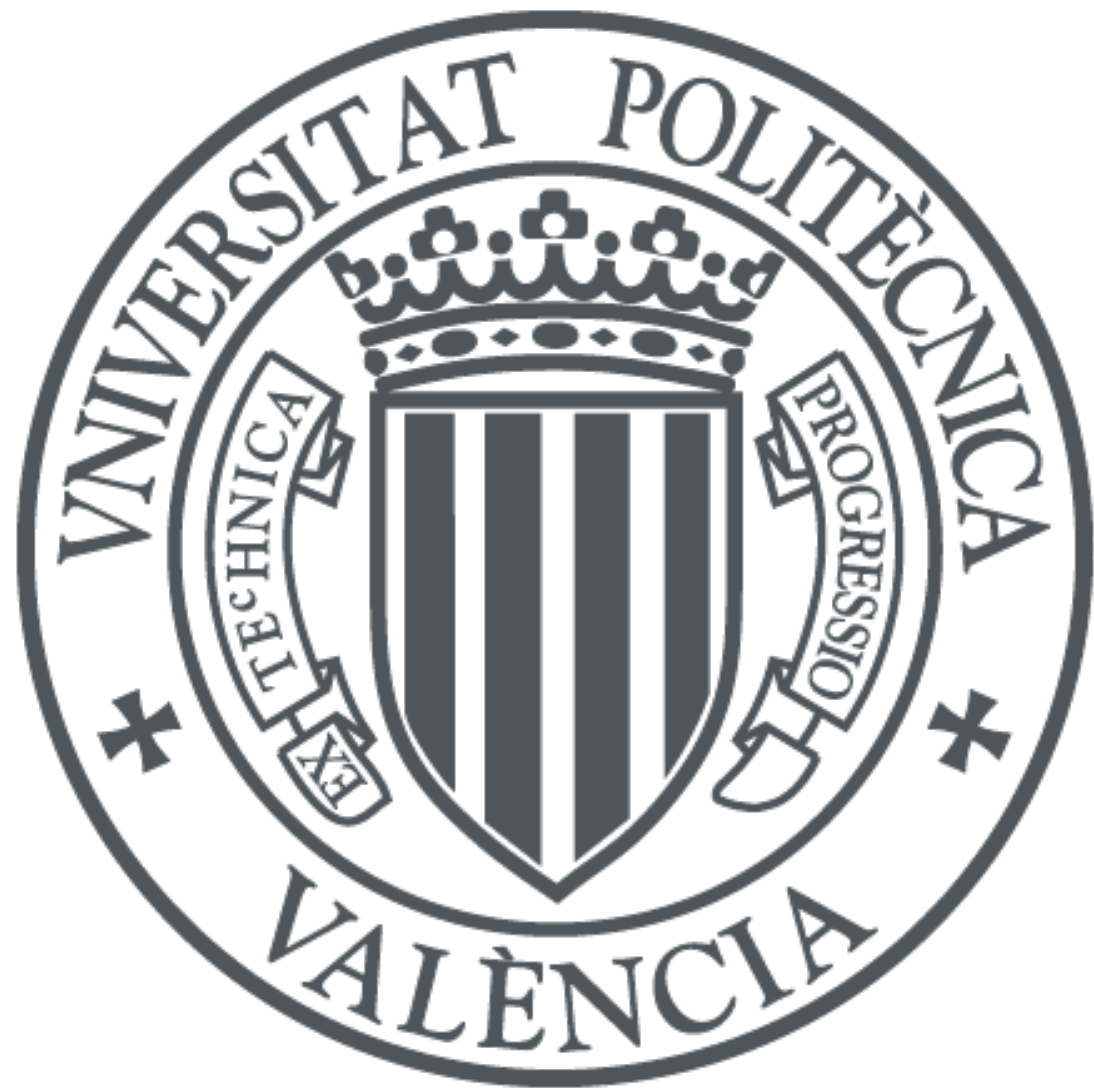

The final publication is available at

http://dx.doi.org/10.1109/TTHZ.2014.2373313

Copyright Institute of Electrical and Electronics Engineers (IEEE)

Additional Information

(c) 2015 IEEE. Personal use of this material is permitted. Permission from IEEE must be obtained for all other users, including reprinting/ republishing this material for advertising or promotional purposes, creating new collective works for resale or redistribution to servers or lists, or reuse of any copyrighted components of this work in other works. 


\title{
All-fiber Centralized Architecture for Parallel Terahertz Sensors
}

\author{
Alexander Bockelt, Jesus Palací, Borja Vidal, Senior Member, IEEE
}

\begin{abstract}
The use of optical fiber for signal distribution and remote operation of a set of parallel inspection units based on THz time domain spectroscopy is presented and analyzed. A centralized approach with optical source-sharing simplifies the implementation and cost of non-destructive inspection platforms, where several sensing units have to work in the same facility, such as factories, security controls, etc. The cost of such a distribution system is evaluated, its feasibility experimentally demonstrated and key features relevant to the system performance are discussed.
\end{abstract}

Index Terms - fiber optics, THz spectrometry, signal transmission lines

\section{INTRODUCTION}

S ENSING instruments exploiting the $\mathrm{THz}$ band $(0.3-10$ $\mathrm{THz}$ ) offer an attractive approach to non-destructive inspection of a wide range of materials with applications in an increasingly extensive variety of industries: in-line monitoring of plastics, compounds, glass, paper, etc.; analytical sciences; biological, pharmaceutical and medical sciences; quality control; environmental monitoring; security, etc. [1-2]. In many cases, these instruments sense features that are not accessible with sensors working in other spectral regions such as the optical, infrared or X-ray bands or complement the information obtained by these. Since $\mathrm{THz}$ radiation is located between the microwave and the optical regions of the electromagnetic spectrum, they exhibit merits of both bands: good penetration depth as well as sub-millimeter resolution. When exposed to terahertz radiation neither scanned objects nor operators run considerable damage or health risk as opposed to X-ray-based inspection systems. Additionally, many materials that are opaque in the visible and near-infrared regime (such as plastics, paper, cardboard, textiles, etc.) are transparent in the $\mathrm{THz}$ region, while many other materials present characteristic spectroscopic fingerprints in this band. Thus, the THz band allows the inspection of materials inside conventional packages.

$\mathrm{THz}$ sources may be broadly classified depending on the generation technologies as: up-conversion from microwave frequencies (which usually provides narrowband radiation) [34], direct $\mathrm{THz}$ generation (for example, using quantum cascade lasers [5-6]) or down-conversion from optical

Alexander Bockelt, Jesús Palací and Borja Vidal are with the Nanophotonics Technology Center, Polytechnic University of Valencia, 46022 Valencia, Spain (e-mail: abockelt@ntc.upv.es; jespalpe@teleco.upv.es; bvidal@depcom.upv.es) frequencies (which can provide either narrow or broadband signals) [1]. Probably the most widely employed optical technique for producing terahertz energy is based on photoconductive switches or antennas (PCAs) [7] due to the tradeoff they offer in terms of emitted power, bandwidth, simplicity and dynamic range.

Several photoconductive materials have been tested for PCAs: low-temperature-grown gallium arsenide (LT-GaAs), radiation-damaged silicon-on-sapphire (RDSOS), chromiumdoped gallium arsenide (Cr:GaAs), indium phosphide (InP) and amorphous silicon. Among these LT-GaAs is probably the most common. The physical generation mechanism begins with an ultra-short laser pulse (commonly emitted by femtosecond-lasers) of sufficiently short wavelengths that are capable of creating free charge carriers in the photoconductor material via photo-excitation. The free carriers then accelerate due to a static bias field building up a transient photocurrent and this time-varying current radiates $\mathrm{THz}$ electromagnetic waves [2]. To provide sufficiently high photon energies to match or exceed the band gap of LT-GaAs, pulsed sources at 780-820 nm as Ti:Sapphire lasers are commonly employed.

Since cost is one of the main limitations to a wider deployment of THz sensors, other materials with smaller band gaps than LT-GaAs have been proposed as photoconductive material. Thus, expensive and bulky Ti:Sapphire lasers can be replaced by low cost $1550 \mathrm{~nm}$ telecom sources. These materials include LT-InGaAs [8], LT-GaAsSb [9], ionimplanted InGaAs [10] and super-lattice structures with LTInGaAs/InAlAs [11-12].

Pulsed THz PCAs based on $1550 \mathrm{~nm}$ sources allow the implementation of all-fiber $\mathrm{THz}$ Time-Domain Spectroscopy (THz-TDS) systems [11-19] rivaling the traditional free-space THz systems. All-fiber implementations are less susceptible to optical misalignment, are less cumbersome and, most notably, show lower cost maintaining good performance. The former takes effect when it comes to precisely coupling the light into the PCAs or if mechanical perturbations (e.g. vibrations in the audio frequency range in an industrial environment) occur. Furthermore, ambient parameters like humidity, dust/vapor concentration, etc. do not affect propagation in fibers, whereas they could cause degradation of an optical beam.

Usually all-fiber THz-TDS systems use very short fiber lengths. However, the excellent features of optical fiber as transmission medium can be further exploited to simplify large $\mathrm{THz}$ systems by establishing a centralized common signal source with several remote emitter/receiver heads [18- 
19]. This approach allows additional cost reduction by sharing the optical source and increases the system flexibility. These schemes could be applied, for example, in in-line quality monitoring of several production lines in a factory, security controls with many parallel security checkpoints as in airports, customs, large characterization laboratories, etc.

In this paper, an analysis of a centralized fiber-based architecture feeding a set of parallel $\mathrm{THz}$ sensing heads is presented. The benefits of this approach as well as the critical parameters are identified and discussed. The paper is organized as follows: In Section II the all-fiber centralized architecture is introduced. Section III discusses the main limitations introduced by the fiber distribution scheme. In Section IV experimental results showing the feasibility of the approach are presented.

\section{Centralized Fiber Distribution ARChitecture}

Optical fiber allows the remote feeding of a set of $\mathrm{THz}$ emitters/receivers thanks to its low transmission loss. Thus, the whole system complexity can be reduced by using a centralized architecture that incorporates simplified modules that ease the measurement setup in industrial scenarios. Centralization also allows simplification of the control system.

The small size and weight, flexibility and robustness of optical fiber facilitates the deployment of the $\mathrm{THz}$ sensing equipment in industries as well as the adaptation of the sensing heads to already deployed machinery. Finally, the immunity to electromagnetic interference of optical fibers simplifies deployment in harsh noisy industrial environments.

Figure 1 shows the proposed architecture for the distribution of femtosecond pulses from a single source to several $\mathrm{THz}$ generation and detection systems. The pulses emitted by a single fs optical source at $1550 \mathrm{~nm}$ are stretched employing a dispersive module. This is necessary to compensate chromatic dispersion. One of the advantages of employing photoconductive antennas fed in the $1550 \mathrm{~nm}$ band is that dispersion compensation becomes simpler.

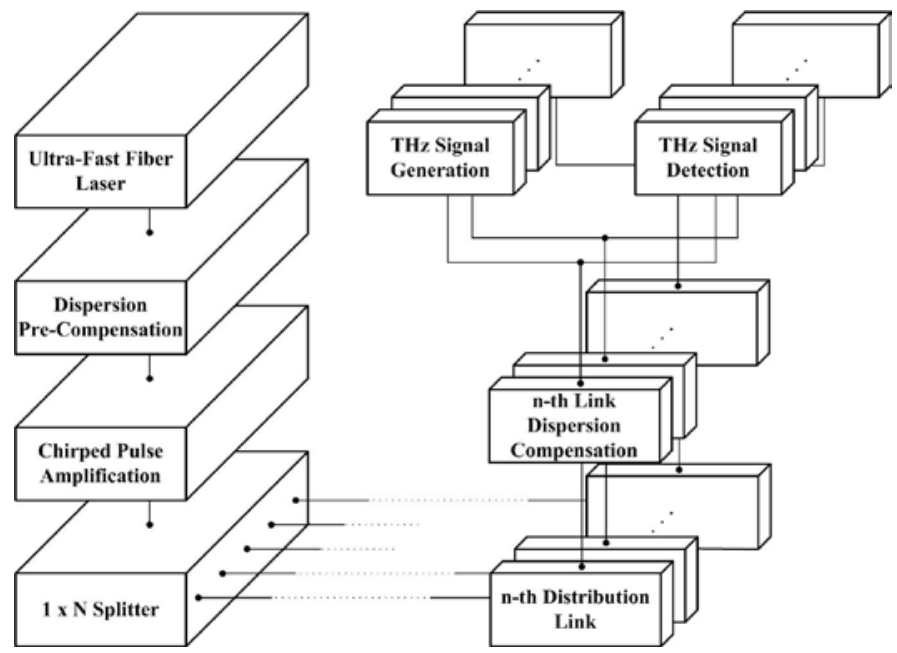

Figure 1. Centralized fiber distribution architecture concept
Low order dispersion terms can be compensated employing dispersion compensation-fibers (DCF) or chirped fiber Bragg gratings developed for the telecom industry.

The stretched pulses are amplified by an erbium-doped fiber amplifier (EDFA) to compensate the distribution loss in the optical splitter. A stretched pulse has significantly lower peak power and thus non-linear effects occurring in the EDFA become less harmful. In particular, self-phase modulation (SPM) has been proved to become decisive for the pulse quality when high optical powers are employed [15]. The amplified optical pulse is then distributed by means of an optical splitter and standard single-mode fiber (SSMF) links to different $\mathrm{THz}$ heads. Each distribution link is made up of a certain amount of SSMF, so that in case of different link lengths an individual dispersion compensation for the respective link had to be implemented.

The femtosecond optical source is a considerable percentage of the spectrometer cost. Therefore, source sharing between several $\mathrm{THz}$ heads can result in a considerable cost reduction. Figure 2 shows an estimation of the cost reduction for a typical all-fiber setup. As it can be seen, a centralized THz-TDS system might reduce the system cost to between $50 \%$ and $70 \%$ of the cost of a set of conventional THz-TDS systems for a number of parallel sensing heads approximately greater than five or higher. The relative cost reduction asymptotically approaches a certain limit within this percentage range, which is given by the cost of the components used to build up the spectrometer. The depicted curves were calculated assuming high and low price limits of conventional commercial telecom laser sources. In a centralized architecture additional sources can be saved, whereas the remaining components' cost increases linearly with the number of inspections units.

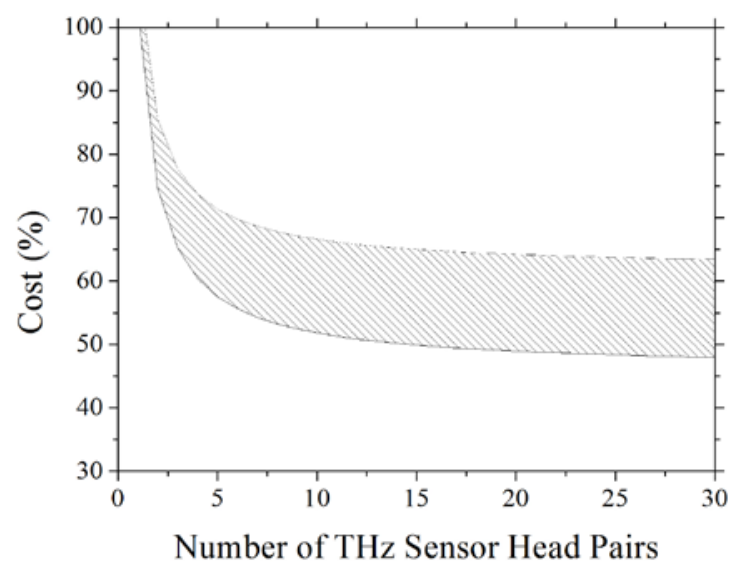

Figure 2. Range of potential cost reduction due to optical source sharing in THz-TDS.

\section{ARCHITECTURE MEASUREMENTS}

To evaluate the feasibility of an all-fiber centralized architecture, the set-up depicted in Figure 3 was implemented in the laboratory. 


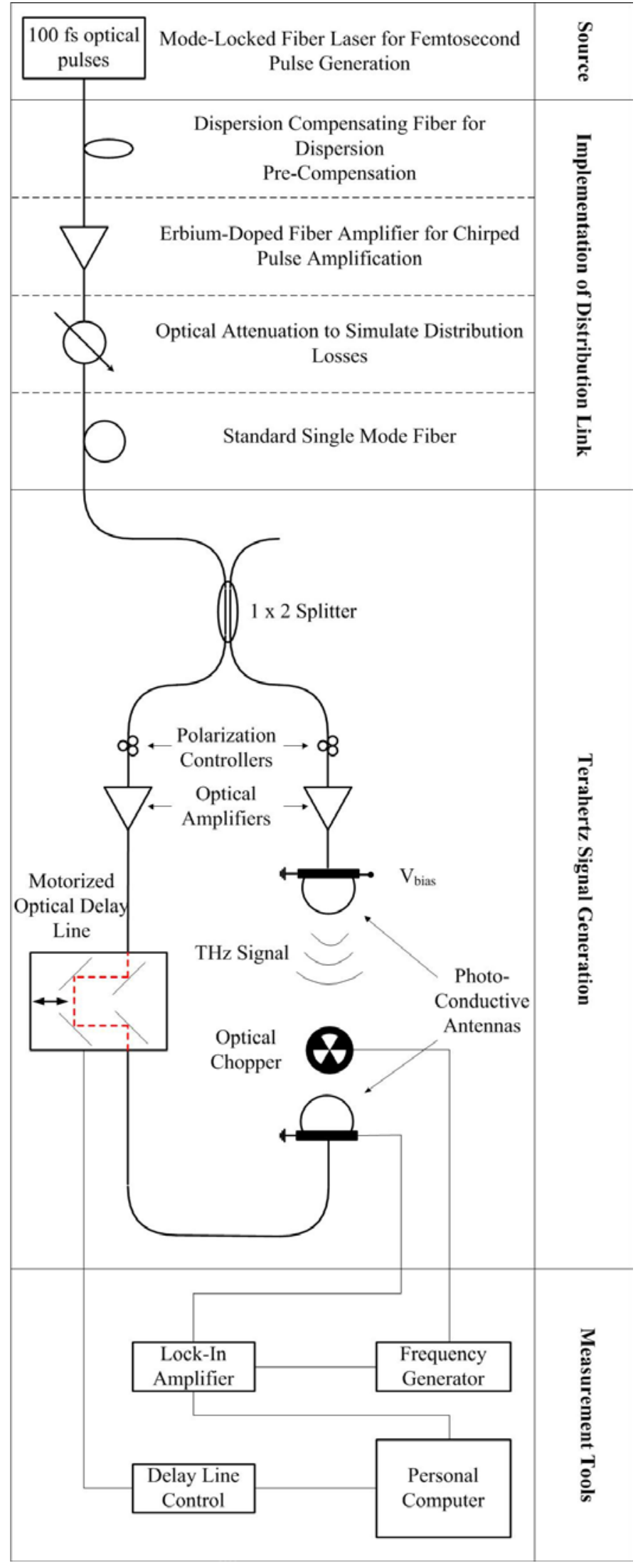

Figure 3. Set-up of the all-fiber distribution link architecture. The performance was compared, when the section "Implementation of Distribution Link" was either inserted or omitted.
The system was designed such that the section, which implements the fiber distribution link, could easily be in- and excluded, to assess how it affects the quality of the terahertz generation and detection process.

Femtosecond optical pulses (100fs) with a mean power of $2.5 \mathrm{~mW}$ and a repetition rate of $50 \mathrm{MHz}$ were stretched by means of a DCF reel with a total dispersion of $-0.33 \mathrm{ps} / \mathrm{nm}$. Stretched pulses subsequently were amplified (chirped pulse amplification, CPA) by an EDFA. Then these amplified pulses should be split for fiber distribution by a $1 \mathrm{xN}$ fiber splitter. However, to facilitate the experimental set-up this splitter was replaced by an optical attenuator (OA), which simulated the splitting loss. An optical loss of $12 \mathrm{~dB}$, corresponding to a $1 \times 16$ splitter, was included in the link. The attenuator was followed by a standard SMF fiber link of $100 \mathrm{~m}$. The delivered pulses were split into two branches and directed to the emitter and the receiver, respectively. Due to the initial dispersion pre-compensation, stretched pulses (>10 ps) are measured at the input of each branch. Consequently the length of the fibers in both branches must be tailored precisely to recompress the pulses to their original shape best possibly. The pulses were passed through polarization controllers (PCs) and two high-output power optical amplifiers (EDFAs) before being injected into InGaAs/InAlAs PCAs. The emitter PCA electrodes were biased with $18 \mathrm{~V}$. When femtosecond optical pulses arrive at the emitter PCA, ultra-short electrical pulses with their principal frequency content in the $\mathrm{THz}$ range are generated and most of its energy is coupled into free-space by means of a high-resistivity hyper-hemispherical silicon lens. This THz signal propagates through free-space to the receiver PCA, where the terahertz signal is sampled by changing the delay of the optical gate pulses arriving at the receiver. The sweeping of the THz pulse was done using a motorized optical delay line (MODL). The THz signal generated by this kind of system is quite noisy, so phase-sensitive detection by a lock-in amplifier (LIA) was employed. Therefore an optical chopper (OC) was modulating the amplitude of the $\mathrm{THz}$ signal at 1 $\mathrm{kHz}$.

Autocorrelation traces of the optical pulses taken at the input of the antennas are shown in Figure 4. It can be appreciated how the optical pulses at the input of the PCAs have been stretched by the distribution link due to residual dispersion ( $a, b)$ compared to those obtained when the fiber distribution section was omitted (c, d). The pulse width or its full width at half maximum (FWHM) is a decisive parameter in $\mathrm{THz}$ generation and detection in PCAs [20].

Figure 5 includes the detected terahertz signal (a) as well as its spectrum (b), where the $10 \mathrm{~dB}$ bandwidth of the Fourier spectrum falls close to $1 \mathrm{THz}$ and signal frequencies up to 2 $\mathrm{THz}$ are observed. For comparison the curves for the system without the distribution link are included. It becomes clear that the system suffers a degradation of the dynamic range due to additional noise introduced by the optical amplifier and a degradation of bandwidth due to the dispersion-broadened optical pulses. Both topics are treated in Section IV. Although the performance has decreased, $\mathrm{THz}$ frequencies up to 1.5 $\mathrm{THz}$ have been obtained for a $100 \mathrm{~m}$ SSMF distribution link. 


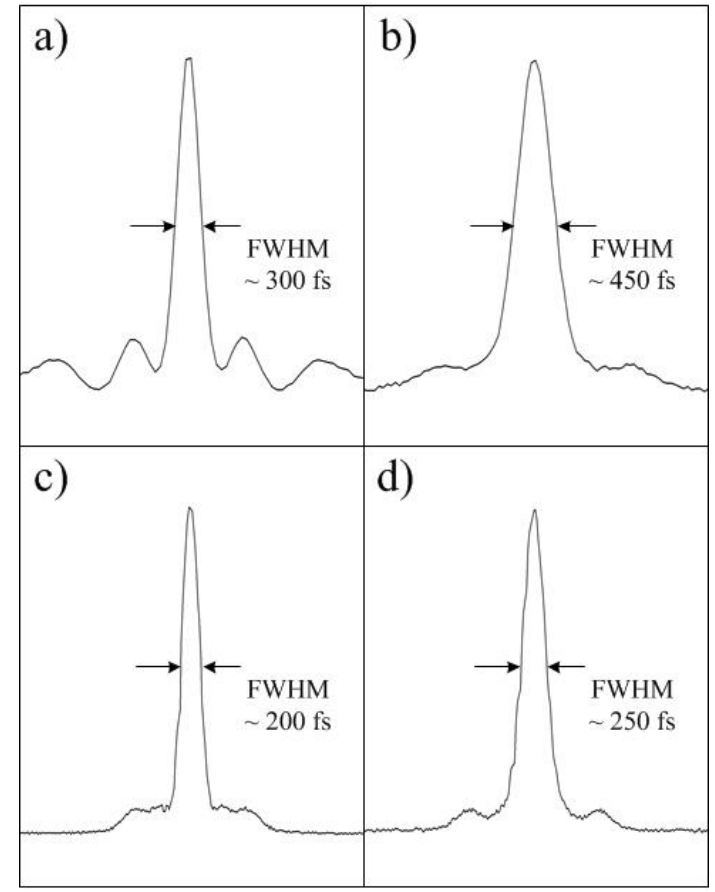

Figure 4. Autocorrelation traces of the optical pulses at the emitter (a, c) and receiver (b, d) PCA's input with (a, b) and without (c, d) distribution link.

a)

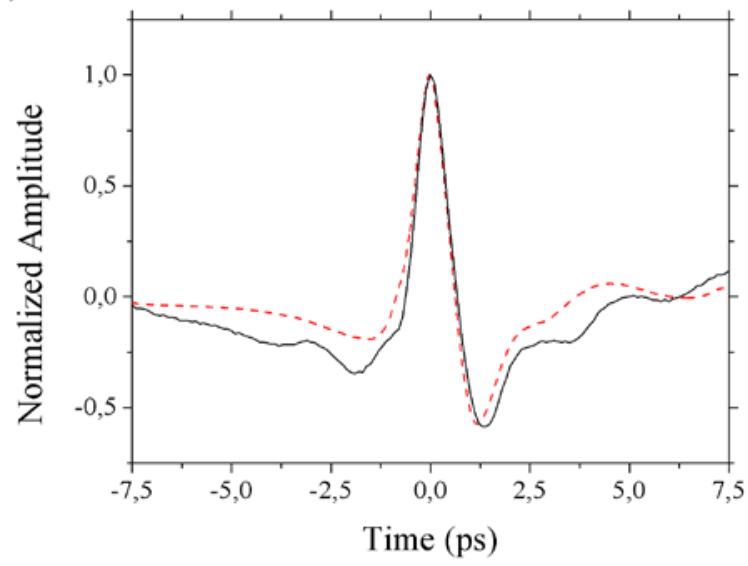

b)

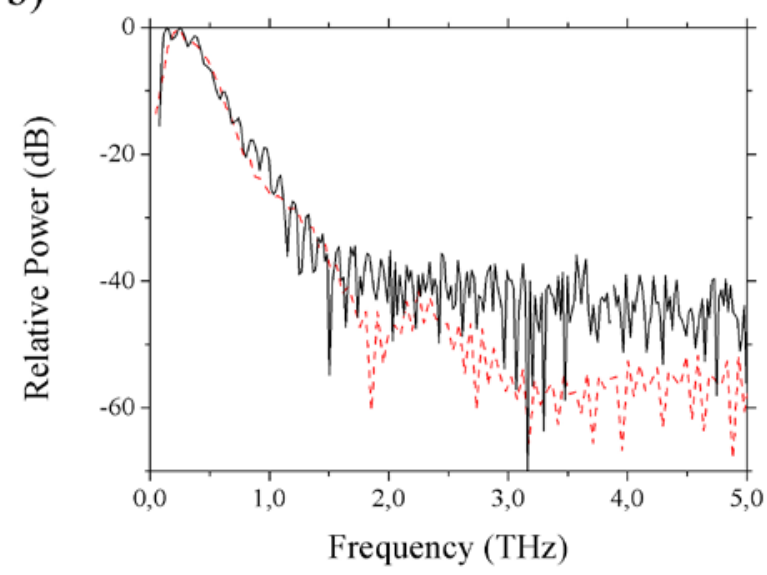

Figure 5. (a) Time-resolved $\mathrm{THz}$ pulses and (b) their corresponding frequency spectra with $100 \mathrm{~m}$ distribution link (solid lines) and without distribution (dashed lines).
The feasibility of long-haul fiber distribution links feeding THz sensing units has also been tested employing an SMF link of $25 \mathrm{~km}$ of length with the corresponding compensation module, using a similar set-up to the one shown in Figure 3. Once more the positive and negative dispersion in both fiber types has to be canceled out precisely to retain narrow pulses. This cancellation becomes gradually more sensitive with increasing fiber length since residual dispersion given by mismatches in higher-order dispersion parameters directly depends on fiber length. Additionally, with longer fiber links, time jitter becomes an issue and actions have to be taken to compensate it. Section IV provides a detailed treatment of these issues. Figure 6 shows the THz spectra obtained with and without the long-haul fiber link. This result shows the feasibility of the approach when limiting factors are addressed.

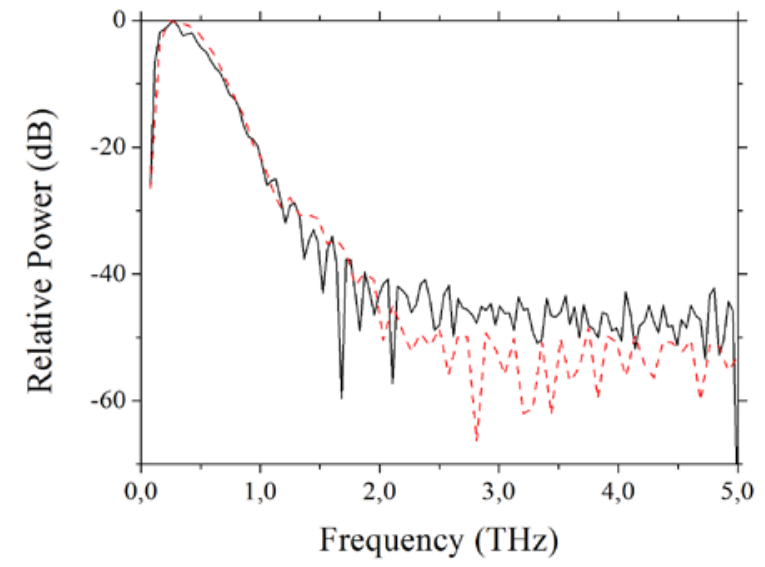

Figure 6. Spectra of $\mathrm{THz}$ pulses with $25 \mathrm{~km}$ distribution link (solid line) and without distribution link (dashed line).

\section{FIBER IMPLEMENTATION FEATURES}

Although fiber-based THz systems using PCAs allow the development of complex centralized architectures not feasible with free-space optics, optical fiber implementations show several critical features that might affect or limit the performance of centralized $\mathrm{THz}$ inspection instruments. In this section the impact of fiber transmission properties on the performance of $\mathrm{THz}$ sensing are particularly studied.

\section{A. Chromatic Dispersion}

Unlike free-space, optical fiber is a dispersive medium. Thus, light propagation in fiber results in severe stretching of femtosecond pulses after considerably short fiber lengths. The broadening factor of an unchirped Gaussian pulse of root mean square (RMS) width $\sigma_{0}$ due to chromatic dispersion is given by (1) [21].

$$
\frac{\sigma(z)}{\sigma_{0}}=\sqrt{1+\left(\frac{\beta_{2} z}{2 \sigma_{0}^{2}}\right)^{2}+0.5\left(\frac{\beta_{3} z}{4 \sigma_{0}^{3}}\right)^{2}}
$$

where $\beta_{2}$ and $\beta_{3}$ are the fiber's second and third order dispersion parameters, respectively, and $z$ is the fiber length. 
Chromatic dispersion has to be accurately compensated to deliver almost transform-limited pulses to the PCAs and maximize the $\mathrm{THz}$ generation and detection. The width of the optical pulses controls the bandwidth of the $\mathrm{THz}$ signals, as shown in (2) [20]:

$$
\overline{I_{r e c}}(f) \propto f e^{-2(\sqrt{2} \pi f \sigma)^{2}} .
$$

where $j_{r e c}$ is the spectrum of the measured photocurrent and $f$ is the frequency.

As discussed in Section II, different approaches are commercially available to compensate second and even third order dispersion terms. Probably the most common method is the use of dispersion compensation fibers. Figure 7 shows an example of the degradation of the $\mathrm{THz}$ spectrum due to residual dispersion, expressed as a length of uncompensated SMF. In particular, the example shown in the figure assumes a fiber path of 100 meters of SMF (dispersion $\mathrm{D}=20.8$ $\mathrm{ps} /(\mathrm{nm} \cdot \mathrm{km})$ and dispersion slope $\left.S=0.061 \mathrm{ps} / \mathrm{nm}^{2} \cdot \mathrm{km}\right)$ plus the length indicated, compensated by a section of 40.895 meters of DCF $(\mathrm{D}=-50.8 \mathrm{ps} /(\mathrm{nm} \cdot \mathrm{km}), \quad \mathrm{S}=-0.154$ $\left.\mathrm{ps} /\left(\mathrm{nm}^{2} \cdot \mathrm{km}\right)\right)$. Due to the broadband nature of femtosecond pulses, care has to be taken to use DCF modules which compensate, at least partially, the third order dispersion term.

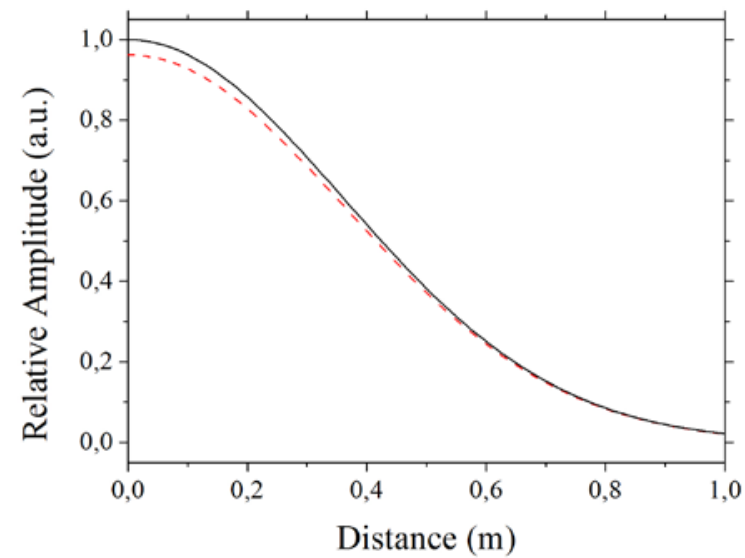

Figure 7. Effect of residual chromatic dispersion on the amplitude at $1 \mathrm{THz}$ of the detected photocurrent. Solid: Effect of second order dispersion. Dashed: Combined effect of second and third order dispersion.

Given the tight constraints of femtosecond pulse fiber distribution, dispersion changes due to temperature variations [20] might affect the THz signal in industrial scenarios. The thermal coefficient of the chromatic dispersion may have opposite signs for fibers with different dispersion sign, so it is possible to partially compensate temperature-induced dispersion drifts by combining different fibers with suitable thermal coefficients. Besides, fibers with low dispersion slopes are more convenient not only in terms of dispersion slope compensation but also to achieve better thermal stability. Figure 8 shows the effect of the dispersion temperature dependence on the $\mathrm{THz}$ signal bandwidth assuming $\mathrm{D}_{\mathrm{SMF}}=20.8$ $\mathrm{ps} /(\mathrm{nm} \cdot \mathrm{km}), D_{\mathrm{DCF}}=-50.8 \mathrm{ps} /(\mathrm{nm} \cdot \mathrm{km})$, no third order dispersion and for two combinations of dispersion thermal coefficients [22]: a) SMF: $-0.0016 \mathrm{ps} /\left(\mathrm{nm} \cdot \mathrm{km} \cdot{ }^{\circ} \mathrm{C}\right)$, DCF: $0.004 \mathrm{ps}$ $/\left(\mathrm{nm} \cdot \mathrm{km} \cdot{ }^{\circ} \mathrm{C}\right)$; b) SMF: $-0.0016 \mathrm{ps} /\left(\mathrm{nm} \cdot \mathrm{km} \cdot{ }^{\circ} \mathrm{C}\right)$; DCF: 0.0009 $\mathrm{ps} /\left(\mathrm{nm} \cdot \mathrm{km} \cdot{ }^{\circ} \mathrm{C}\right)$. As can be seen from Figure 8 , temperature dependence might have a considerable impact on the $\mathrm{THz}$ system if the compensating fiber is not suitably chosen.

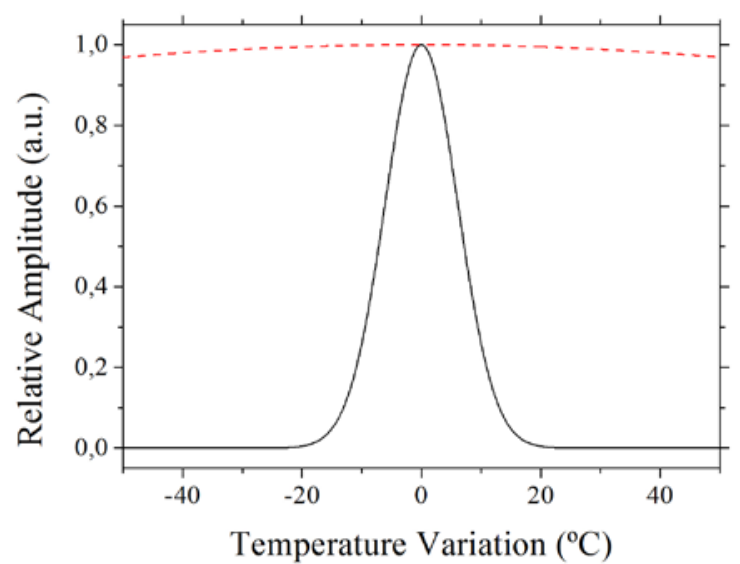

Figure 8. Effect of the temperature dependence of chromatic dispersion on the relative amplitude at $1 \mathrm{THz}$ of the detected photocurrent. a) SMF: -0.0016 $\mathrm{ps} /\left(\mathrm{nm} \cdot \mathrm{km} \cdot{ }^{\circ} \mathrm{C}\right)$; DCF: $0.004 \mathrm{ps} /\left(\mathrm{nm} \cdot \mathrm{km} \cdot{ }^{\circ} \mathrm{C}\right.$ ) (solid); b) SMF: -0.0016 $\mathrm{ps} /\left(\mathrm{nm} \cdot \mathrm{km} \cdot{ }^{\circ} \mathrm{C}\right)$; DCF: $0.0009 \mathrm{ps} /\left(\mathrm{nm} \cdot \mathrm{km} \cdot{ }^{\circ} \mathrm{C}\right)$ (dashed).

\section{B. Nonlinear Effects}

Nonlinear effects in optical fiber can result in severe distortion of the femtosecond pulses when high optical power is used [15]. As an example, in [22] Self-Phase Modulation (SPM) was used in combination with third-order dispersion, for $\mathrm{THz}$ pulse shaping. These distortions are in general detrimental to the generation and detection of $\mathrm{THz}$ signals in PCAs and mainly result in severe broadening of the gate pulses, which leads to the lowering of bandwidth. However, distortion by nonlinear effects can be considerably reduced by using suitable optical fibers and keeping low peak amplitudes through pulse stretching, as done in chirped pulse amplification.

\section{Fiber Joint Impairments}

The different fiber pigtails of the components of the centralized $\mathrm{THz}$ architecture have to be joined. This can mainly be done using fiber connectors, fusion splices, or semipermanent splices. Fusion splices offer low attenuation and return loss but at the cost of reducing reconfiguration capability. They might be the best option for inspection platforms in an industrial scenario, since usually once the system is deployed it does not experience frequent needs for extensions.

If fiber connectors are used to keep reconfigurability, care has to be taken to reduce connector loss. As it is shown in Figure 9, a considerable portion of optical power and thus $\mathrm{THz}$ amplitude might be lost through reflections at air gap interfaces in fiber junctions. In the test set-up the amplitudes tripled when a refractive index matching gel to close these gaps was employed. 


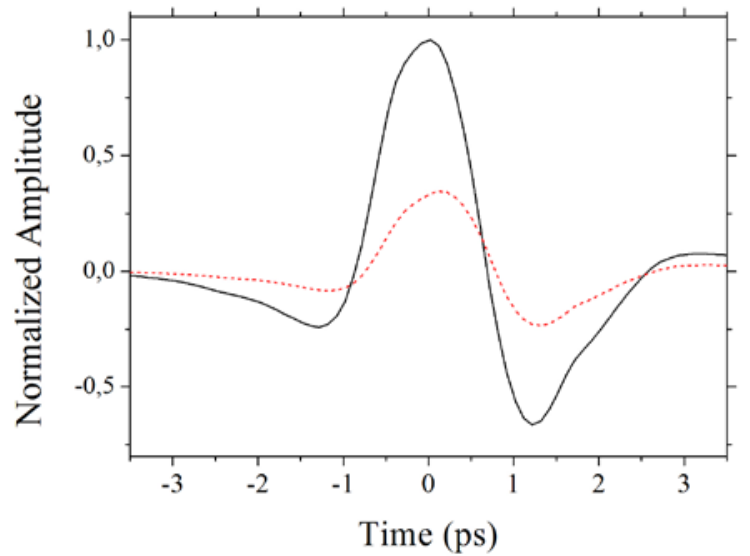

Figure 9. Influence of connector gaps: Use of index matching gel (solid line) and pulse suffering from connector gaps/reflections (dashed line)

\section{Time Delay Jitter}

Like every transmission medium, optical fiber shows fluctuations of the propagation delay. So, in addition to the optical source jitter, there are timing errors caused by the influence of thermal, acoustic, and mechanical disturbances on the optical fiber that result in fluctuations on the output pulse arrival time. For example, typical commercial fibers for optical communications show thermal coefficients of the propagation delay around $38 \mathrm{ps} /(\mathrm{km} \cdot \mathrm{K})$ [23].

This change in time of flight of the optical pulses limits the maximum fiber length and the temperature operation range of the $\mathrm{THz}$ architecture. In a conventional THz-TDS system, where $\mathrm{THz}$ pulses are scanned in a few ps time window, unstabilized operation can be performed with a simple control on the MODL. However, faster inspection systems, e.g. [24], would require active stabilization methods [23, 25-26], which can provide long term timing stability with up to subfemtosecond precision [26].

\section{E. Polarization}

It is well-known that light polarization at the output of a long fiber section changes randomly over time [27]. To investigate the effect of polarization changes on the LTInGaAs/InAlAs PCAs, a polarization controller was inserted in the fiber path. While the polarization was being changed continuously by a polarization controller to cover most states of polarization, the relative error of the $\mathrm{THz}$ pulse amplitude was measured to be 0.0066 . For comparison the same was performed without actively changing the polarization obtaining a relative error of 0.0028 . It was observed that the relative error, calculated by making use of a temporal standard deviation, roughly doubles when the polarization was changed. However, the optical power reaching the $\mathrm{THz}$ emitter was assessed by the same method and it turned out that its standard deviation and relative error also double. It is assumed therefore that $\mathrm{THz}$ amplitude fluctuations were due to amplitude variations. Concluding, there is no harmful effect of polarization changes that deteriorates the measured $\mathrm{THz}$ amplitudes and degrades the spectroscopy performance. Nevertheless, long-term polarization changes will provoke time jitter due to polarization mode dispersion (PMD) and contribute to the timing errors described in Section IV.D.

Another issue to be taken into account is residual differential group delay (DGD). It gets stronger as the fiber path gets longer. To assess DGD effect on THz generation a SMF patch cord was replaced by a polarization maintaining fiber (PMF), which shows a significantly larger DGD. Depending on the incoming polarization the pulses reach the antennas with or without delay, while in most of the cases, when both polarization components are present, two pulses emerge. This superposition leads to notch filtering in the frequency domain. Figure 10 shows the temporal traces of pulses with different polarizations (a) and the corresponding spectra (b). For systems with long fiber lengths systems it is thus recommendable to employ polarization maintaining fiber in the whole setup to avoid polarization induced changes and improve system stability in terms of amplitude and jitter.

a)

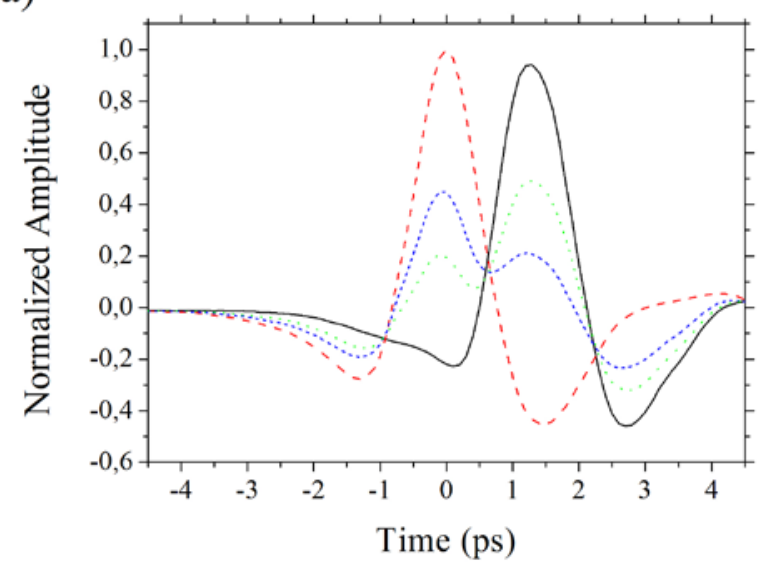

b)

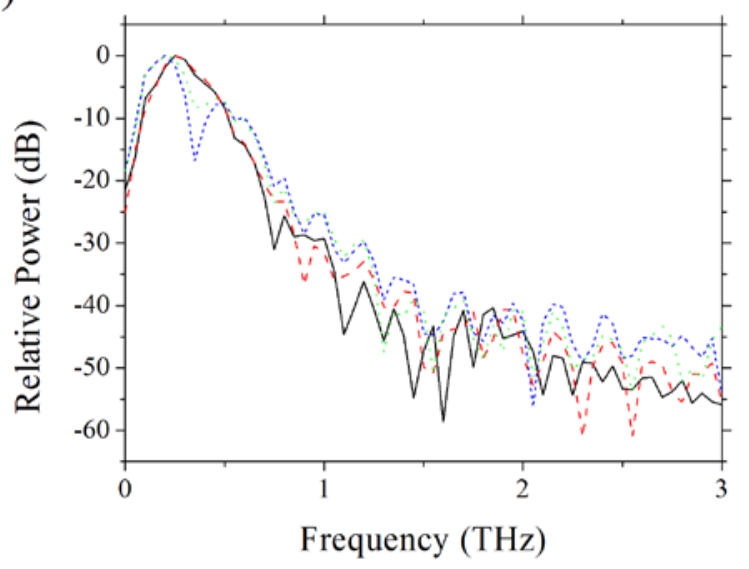

Figure 10 a) Pulse forms/delays due to polarization mode dispersion and b) corresponding spectra

\section{F. Additional Noise Sources}

The use of EDFAs in the centralized $\mathrm{THz}$ system adds a high degree of flexibility in the system and allows the 
distribution of the signal in many parallel links, improving the cost efficiency of the system. However, EDFAs introduce amplified spontaneous emission (ASE) noise to the $\mathrm{THz}$ emitters/receivers.

To assess the effect of the use of EDFAs in $\mathrm{THz}$ sensing systems, a THz-TDS system has been modified to include a controlled ASE source. A piece of SSMF has been replaced by a fiber coupler to enable the injection of an additional signal from an ASE source to either the emitter or the receiver antenna. Figure 11a) shows the effect on the amplitude of the pulses when injecting different ASE levels into the emitter and the receiver, whereas $b$ ) compares the corresponding dynamic ranges. As can be seen, ASE noise reduces the amplitude of the THz signal, as shown in [28], for an additional continuous wave signal. This reduction in the amplitude of the $\mathrm{THz}$ pulses results in a degradation of the dynamic range although the ASE power levels considered here are considerably higher than expected values in a practical configuration. Low ASE noise levels reduce the amplitude almost negligibly and thus cause the dynamic range only to suffer minor degradations. Nevertheless this effect can further be reduced by using a preamplifier configuration.

a)

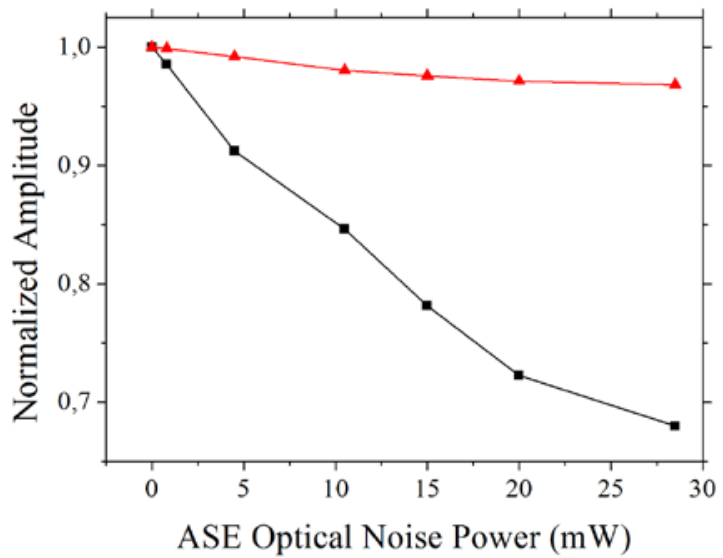

b)

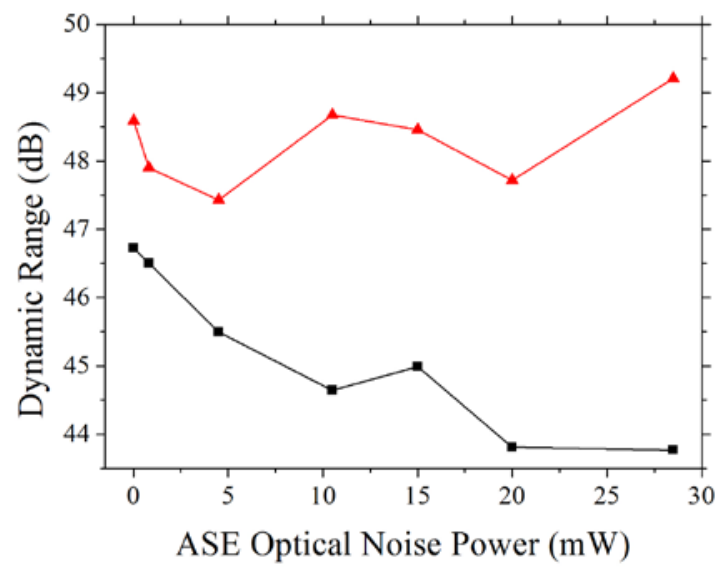

Figure 11 Effect of the injection of ASE noise into the emitter antenna (triangles) and receiver antenna (squares), in terms of amplitude of the $\mathrm{THz}$ pulses (a) and dynamic range (b).
Additionally, ASE noise can induce time jitter which will be combined with jitter sources commented in Section IV.D. For an input Gaussian pulse and the fiber lengths considered in this work (100 m and $25 \mathrm{~km}$ ), ASE-noise induced time jitter contribution would be of the order of 0.1 fs and 15 fs, respectively, according to estimations obtained from simulations carried out using VPI Photonics software.

\section{CONCLUSION}

A centralized fiber-architecture has been presented that allows the parallel operation of various $\mathrm{THz}$ spectrometers or sensors. In a similar way as in optical centralized schemes in communication systems, such as Passive Optical Networks (PON), Radio over Fiber (RoF), etc., fiber can be exploited to reduce the cost of spectroscopy instruments in applications where a set of sensing units are required.

In this paper the feasibility and performance of this approach was analyzed. The use of robust optical fiber distribution links eases deployment in industrial scenarios, while source sharing permits reduction of the total cost of up to $50 \%$. Limiting effects and parameters such as chromatic dispersion, nonlinear effects in fiber, fiber joint impairments, time delay jitter, polarization and ASE noise were investigated. The most crucial parameter affecting the system performance is the residual dispersion resulting from inaccurate dispersion compensation as well as temperature variations. Precise dispersion compensation is particularly desirable for the delivery of narrow optical pulses required for the $\mathrm{THz}$ generation and detection process. This work shows that besides conventional advantages, like remote and accurate feeding of PCAs and overall robust implementations of THzsensing systems, a centralized fiber architecture is further capable to reduce the cost and complexity and ease the control of $\mathrm{THz}$ sensing solutions.

\section{ACKNOWLEDGMENT}

This work was partially supported by the Spanish Ministry of Economy and Competitiveness through project TEC201235797 and FPU grant 12/02847.

\section{REFERENCES}

[1] M. Tonouchi, "Cutting-edge terahertz technology", Nature Photonics, vol. 1, pp. 97-105, February 2007.

[2] P.U. Jepsen, D. G. Cooke, M. Koch, "Terahertz spectroscopy and imaging - Modern techniques and applications”, Laser \& Photonics Reviews, vol. 5, no. 1, pp. 124-166, 2011.

[3] P.H. Siegel, "Terahertz Technology", IEEE Transactions on Microwave Theory and Techniques, vol. 50, no. 3, pp. 910-928, March 2002.

[4] N. Jun-ichi, P. Piotr, M. Hiroki, T. Kurabayashi, "GaAs TUNNETT diodes oscillating at $430-655 \mathrm{GHz}$ in CW fundamental mode”, IEEE Microwave Wireless Components Letters, vol. 15, no. 9, pp. 597-599,

[5] R. Köhler, A. Tredicucci, F. Beltram, H.E. Beere, E.H. Linfield, A. Giles Davies, D.A. Ritchie, R.C. Iotti, F. Rossi, "Terahertz semiconductor-heterostructue laser", Nature, vol. 417, pp. 156159, May 2002.

[6] G. Scalari, C. Walther, M. Fischer, R. Terazzi, H. Beere, D. Ritchie, J. Faist, "THz and sub-THz quantum cascade lasers", Laser \& Photonics Review, pp. 1-22, 2008. 
[7] D.H. Auston, "Picosecond Optoelectronic Switching and Gating in Silicon”, Applied Physical Letters, vol. 326, no. 3, pp. 101-103, February 1975.

[8] C. Baker, I.S. Gregory, W.R. Tribe, I.V. Bradley, M.J. Evans, E.H. Linfield, M. Missous, "Highly resistive annealed lowtemperature-grown InGaAs with sub-500 fs carrier lifetimes", Applied Physics Letters, vol. 85, 4965, 2004.

[9] J. Sigmund, C. Sydlo, H.L. Hartnagel, N. Benker, H. Fuess, F. Rutz, T. Kleine-Ostmann, M. Koch, "Structure investigation of low-temperature-grown GaAsSb, a material for photoconductive terahertz antennas”, Applied Physics Letters, vol. 87, 252103, 2005.

[10] M. Suzuki, M. Tonouchi, “Fe-implanted InGaAs photoconductive terahertz detectors triggered by $1.56 \mu \mathrm{m}$ femtosecond optical pulses”, Applied Physics Letters, vol. 86, 163504, 2005.

[11] B. Sartorius, H. Roehle, H. Künzel, J. Böttcher, M. Schlak, D. Santze, H. Venghaus, M. Schell, "All-fiber terahertz time-domain spectrometer operating at 1.5 micron telecom wavelengths”, Optics Express, vol. 16, pp. 9565-9570, 2008.

[12] H. Roehle, R.J.B. Dietz, H.J. Hensel, J. Böttcher, H. Künzel, D. Sanze, M. Schell, B. Sartorius, "Next generation $1.5 \mu \mathrm{m}$ terahertz antennas: mesa-structuring of InGaAs/InAlAs photoconductive layers”, Optics Express, vol. 18, no. 3, pp. 2296-2301, 2010.

[13] B. Sartorius, M. Schlak, D. Stanze, H. Roehle, H. Künzel, D. Schmidt, H.-G. Bach, R. Kunkel, and M. Schell, "Continuous wave terahertz systems exploiting 1.5 micrometer telecom technologies”, Optics Express, vol. 17, no. 17, pp. 15001-15007, 2009

[14] H. Roehle, R. Dietz, B. Sartorius, and M. Schell, "Fiber-Coupled Terahertz TDS Combining High Speed Operation with Superior Dynamic Range”, 37th International Conference on Infrared, Millimeter and Terahertz Waves (IRMMW-THz), Wollongong (Australia), 23-28 September 2012

[15] F. Eichhorn, R.K. Olsson, J.C.D. Buron, L. Grüner-Nielsen, J. E. Pedersen, P.U. Jepsen, "Optical fiber link for transmission of 1-nJ femtosecond laser pulses at 1550 nm”, Optics Express, vol. 18, pp. 6978-687, 2010.

[16] Sang-Pil Han, Hyunsung Ko, Namje Kim, Han-Cheol Ryu, Chul Wook Lee, Young Ahn Leem, Donghun Lee, Min Yong Jeon, Sam Kyu Noh, Hyang Sook Chun, and Kyung Hyun Park, "Optical fiber-coupled InGaAs-based terahertz time-domain spectroscopy system”, Optics Letters, Vol. 36, No. 16, pp. 3094-3096, 2011

[17] G. Ducournau, A. Beck, K. Blary, E. Peytavit, M. Zaknoune, T. Akalin, J.-F. Lampin, M. Martin, and J. Mangeney, "All-fiber Continuous Wave Coherent Homodyne Terahertz Spectrometer Operating at 1.55 Micrometer Wavelengths”, 37th International Conference on Infrared, Millimeter and Terahertz Waves (IRMMW-THz), Busan (South Korea), 21-25 September 2009

[18] J. Palací, B. Vidal, "Distributed THz Transmitter/Receiver based on a 1.5 $\mu \mathrm{m}$ Fiber Link", 36th International Conference on Infrared, Millimeter and Terahertz Waves (IRMMW-THz), Houston (USA), 2-7 October 2011.

[19] J. Palací, A.S. Bockelt, B. Vidal, “Amplified Optical Fiber Link for Remote Generation of $\mathrm{THz}$ Radiation”, IEEE International Topical Meeting on Microwave Photonics (MWP2013), Alexandria (USA), 28-31 October 2013.

[20] L. Duvillaret, F. Garet, J.F. Roux, J.L. Coutaz, “Analytical Modeling and Optimization of Terahertz Time-Domain Spectroscopy Experiments Using Photoswitches as Antennas”, IEEE J. of Selected Topics in Quantum Electron., vol. 7, no. 4, pp. 615- 623, July/August 2001.

[21] M.J. Hamp, J. Wright, M. Hubbard, B. Brimacombe, "Investigation into the Temperature Dependence of Chromatic Dispersion in Optical Fiber”, IEEE Photon. Technol. Lett., vol. 14, no. 11, pp. 1524-1526, Nov. 2002.

[22] J. Palací, B. Vidal, "Terahertz Radiation Shaping based on Third-Order Dispersion and Self-Phase Modulation in Standard Single-mode Optical Fiber”, Journal of Infrared, Millimeter and Terahertz Waves, vol. 33, no. 6, pp. 605-614, 2012.

[23] P. Krehlik, Ł. Śliwczyński, Ł. Buczek, M. Lipiński, "Fiber-Optic Joint Time and Frequency Transfer With Active Stabilization of the Propagation Delay”, IEEE Transactions on Instrumentation and Measurement, ol. 61, no. 10, pp. 2844-2851, Oct. 2012.

[24] H. Hoshina, Y. Sasaki, A. Hayashi, C. Otani, "Noninvasive Mail Inspection System with Terahertz Radiation”, Applied Spectroscopy, vol. 63, no. 1, 2009.
[25] J. Kim, J.A. Cox, J. Chen, F.X. Kärtner, "Drift-free femtosecond timing synchronization of remote optical and microwave sources”, Nature Photonics, vol. 2, pp. 733-736, Dec. 2008.

[26] M.Y. Peng, P.T. Callahan, A.H. Nejadmalayeri, S. Valente, M. Xing, L. Güner-Nielsen, E. M . Monberg, M. Yan, J.M. Fini, F.X. Kärtner, "Long-term stable sub-femtosecond timing distribution via a $.2 \mathrm{~km}$ polarization maintaining fiber link approaching 10-21 link stability”, Optics Express, vol. 21, no. 17, Aug. 2013.

[27] G.P. Agrawal, Nonlinear Fiber Optics, Ed. Wiley, 2001.

[28] A. Bockelt, J. Palací, B. Vidal, "Control of terahertz emission in photoconductive antennas through an additional optical continuous wave”, Optics Letters, vol. 38, no. 17, pp. 3123-3125, Aug. 2013

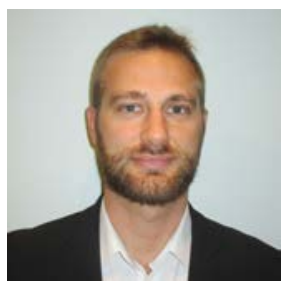

Alexander Bockelt was born in Schweinfurt, Germany, in 1984. He received the Master degree in Physics $(\mathrm{MPh})$ from the Heriot-Watt University of Edinburgh, United Kingdom, in 2008 and the Diploma degree in Physics from the Julius-Maximilians University, Würzburg, Germany in 2011. He realized his diploma thesis at Nanoplus GmbH in Gerbrunn, Germany working on semiconductor lasers based on InP substrates. Currently he is realizing his $\mathrm{PhD}$ thesis forming part of the Terahertz Photonics Group at the Nanophotonics Technology Center of the Universitat Politècnica de València, Spain.

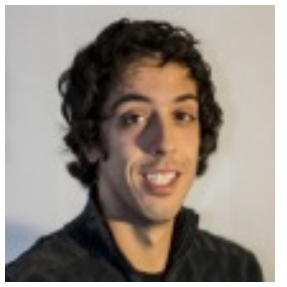

Jesús Palací López was born in Valencia, Spain, in 1985. He received the M.S. and PhD degrees, both in Electrical Engineering, from the Universitat Politècnica de València in 2008 and 2013, respectively. He works as a senior researcher for the Valencia Nanophotonics Technology Center, Spain. His interests include microwave and terahertz technology as well as the study of nonlinear optics and its applications using both fiber and integrated technology.

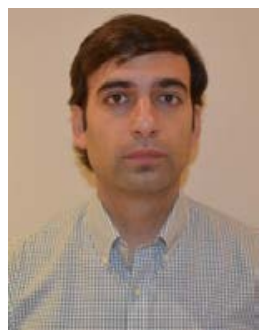

$\begin{array}{lcc}\text { Borja } & \text { Vidal } & \text { (S'99-M'06-SM'13) } \\ \text { received } & \text { the } & \text { Ingeniero de }\end{array}$ Telecomunicación (M.Sc.) and Doctor Ingeniero de Telecomunicación (PhD) degrees from the Universitat Politècnica de València, Spain, in 2001 and 2004, respectively.

In 2005, he joined the Communications Department of the Universitat Politècnica de València, Spain, where he is currently an Associate Professor. In 2010, he was a Visiting Scholar at the University of Kent, UK. His research interest includes $\mathrm{THz}$ photonics, microwave photonics, optical signal processing and electromagnetic sensing. He is the author of more than 50 research articles, 45 contributions to international conferences and 2 patents.

Dr. Vidal received the IEEE Lasers and Electro-Optics Society (LEOS) Graduate Student Fellowship in 2004. He has served as member of the Technical Program Committee of the International Topical Meeting on Microwave Photonics (MWP). 